Submission ID: 43850

\title{
Curvilinear Spectral Elements Application for Sonic Logging Forward
} Modeling

D.I. Sabitov (Skolkovo Institute of Science and Technology), M. Charara (Skolkovo Institute of Science and Technology), A.A. Dotsenko* (Skolkovo Institute of Science and Technology)

\section{SUMMARY}

The interpretation of sonic well logging data is a challenging problem for researches in the oil industry. A fast and accurate numerical method is needed to get the cleat picture of the physics of wave propagation in complex geological surroundings with the presence of strong discontinuity of fluid-solid interface. The Spectral Element modeling allows to solve efficiently the 3D dynamic wave propagation problems with complex physics. For sonic logging problems together with appropriate choice of spatial mesh step size the correct approximation of borehole geometry affects the accuracy of the numerical solution. The goal of this work is to extend the spectral element method to deal with the curvilinear elements and to show its addition value for the mesh construction, approximation of the geometry and increasing the accuracy of numerical solutions. 


\section{Применение криволинейных спектральных элементов для моделирования прямых задач акустического каротажа}

\section{Д.И. Сабитов, М. Шарара,А.А. Доценко* (Сколковский институт науки и технологий)}

\section{Введение}

Анализ и интерпретация данных акустического каротажа является сложной задачей в геофизических исследованиях нефтегазоносных скважин. Для ее решения необходимо с высокой точностью моделировать процесс распространения упругих волн в присутствии интерфейса между жидкостью и твердым телом, учитывать анизотропию и вязкоупругость горных пород, а также особенности геометрии скважин и околоскважинной зоны. Численный метод спектральных элементов (SEM) позволяет эффективно решать трехмерные волновые динамические задачи для неоднородных сред со сложной физикой. Для задач акустического каротажа, помимо выбора шага сетки, существенноевлияние на точность получаемого решения оказывает корректноесеточное описание геометрической формы скважины. Целью данной работы является описание методики использования криволинейных спектральных элементов и иллюстрация её преимуществ при построении геометрии расчетной области, повышения точности решения и ускорения расчетов.

\section{Постановка задачи}

Рассмотрим модельную задачу о распространении волн при проведении акустического каротажа.Цилиндрическая скважина, заполненная жидкой средой, окружена цилиндром большего диаметра, соответствующимупругой горной породе. Монопольный источник акустического сигнала на центральной оси скважины генерирует упругие волны, распространяющиеся в породе и в скважине, отклик системы регистрируется кольцевыми азимутальными приемниками, симметрично расположенными вдоль оси скважины(Горбачев 1990). Для упрощения постановки будем считать, что физический прибор в скважине отсутствует, а источник и приемники являются математическими точками возбуждения и регистрации сигналов (Рисунок 1).

Волновые процессы в упругом материале с плотностью $\rho$ и упругим тензором Сописываются уравнениями эластики для трех компонент вектора перемещений $\vec{u}$ :

$$
\begin{aligned}
& \rho \vec{u}=\nabla \cdot \sigma, \text { уравнения движения, } \\
& \sigma=C: \varepsilon, \varepsilon=\frac{1}{2}\left[\nabla u+(\nabla u)^{T}\right], \text { определяющие соотношения для закона Гука }
\end{aligned}
$$

В жидкой среде с плотностью $\rho_{f}$ и коэффициентом объемного сжатия $K_{f}$ достаточно использовать одно скалярное акустическое уравнение, описывающее эволюцию потенциала $\Phi$, с источником акустического сигнала $f=f(x, t)$ :

$$
\rho_{f} \ddot{\Phi}=\nabla \cdot\left(K_{f} \nabla \Phi\right)+f \text {. }
$$

Граница между жидкостью и упругим телом в данном случае криволинейная с нормалью $\vec{n}$. На ней используются краевые условия связи между двумя типами уравнений:

$$
\begin{aligned}
& \rho_{f} \ddot{\Phi} \cdot \vec{n}=\sigma \cdot \vec{n} \text {, условие непрерывности нормальных напряжений и давления, } \\
& \frac{d}{d \vec{n}} \Phi=\vec{u} \cdot \vec{n}, \text { непрерывность нормальных перемещений в жидкости и упругом теле. (5) }
\end{aligned}
$$

Следуя формализму метода Галеркина, определим неизвестные искомые функции $\vec{u}$, Фчерезлинейные комбинации базисных функций $N_{k}$ с неизвестными коэффициентами $\widehat{\vec{u}}=$ $\sum_{k} \vec{u}_{k} \cdot N_{k}, \widehat{\Phi}=\sum_{k} \Phi_{k} \cdot N_{k}$ и подставим их в уравнения (1-3). Умножая полученные выражения поочередно на базисные функции и интегрируя по вычислительной области $\Omega$, получим систему обыкновенных дифференциальных уравнений: 
$M \frac{\partial^{2} \widehat{\vec{u}}}{\partial t^{2}}+K(\widehat{\vec{u}})-g=0$, где

$M=\int_{\Omega} N^{T} \rho N d \Omega-$ матрица масс,

$K(\hat{\vec{u}})=\int_{\Omega} \nabla N^{T} \cdot \sigma d \Omega-$ матрица жесткости,

$g=\int_{\Gamma} N^{T} \vec{P} d \gamma+\int_{\Omega} N^{T} f d \Omega-$ член, отвечающий за граничные условия и источник. (9) Уравнения для (3) получаются таким же способом. Для интегрирования по времени используется схема Ньюмарка(ZienkevichandTaylorR.F 2000).

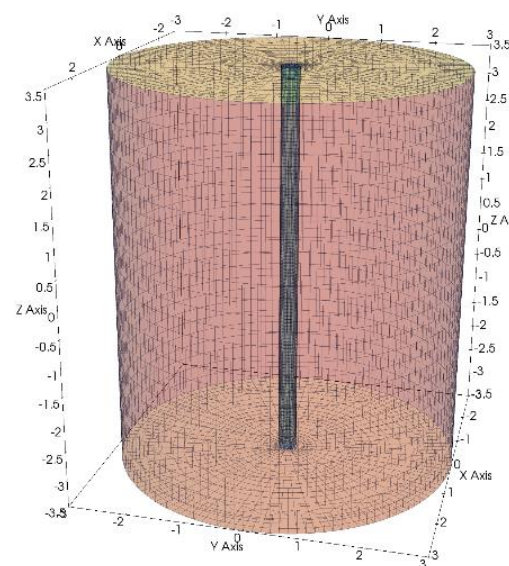

a)

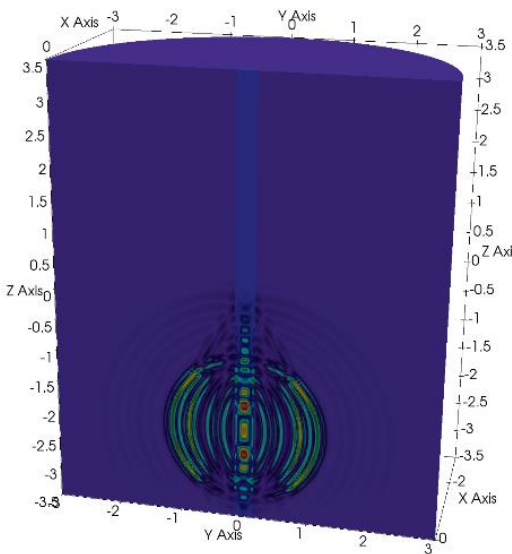

b)

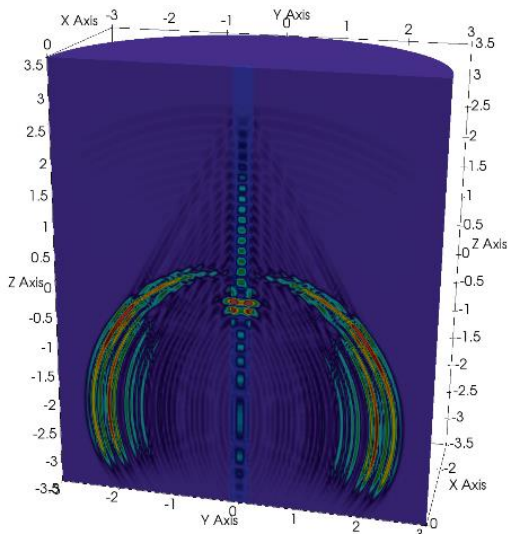

c)

Рисунок 1a) - сеточная модель скважины и окружающей ее горной породы; $b$ ), c)- развитие во времени волновой картины от источника в скважине.

Метод спектральных элементов порядка Rзаключается в использовании квадратурной формулы Гаусса-Лежандра-Лобатто (GLL)для вычисления интегралов (7-9) и специальном построении системы базисных функций-многочленов $N_{k}$.Квадратурная формула состоит из набора $R$ координат узлов и весов на отрезке $[-1 ; 1]$ ипозволяет приближенно вычислять интегралы как сумму значений подынтегральной функции в узлах, умноженных на соответствующий вес: $\int_{-1}^{1} f(x) d x=\sum_{i=1}^{R} f\left(x_{i}\right) \omega_{i}$. В двух- и трехмерном пространствах кубатурная формула строится как прямое произведение одномерных квадратур, что приводит к использованию элементов- топологических четырехугольников или гексаэдров соответственно. Набор базисных функцийна элементе строится из прямого произведения многочленов Лагранжа над узлами GLL таких, что многочлен равен 1 в одном из узлов и 0 во всех остальных.Система таким образом построенных базисных функций обладает свойством дискретной ортогональности(Komatitschetal. 2000), что позволяет существенно уменьшить количество ненулевых элементов в матрице жесткости (8) по сравнению с классическим методом конечных элементов высокого порядка (FEM). При этом порядок аппроксимации метода будет равен количеству узлов $R$ во взятой за основу квадратуре GLL, и погрешность метода при повышении порядка будет экспоненциально убывать. Дискретно ортогональные базисные функции приводят к построению блочно-диагональной матрицы масс (7), тем самым метод SEMиспользует явную схему интегрирования по времени уравнений (6), что дает уменьшение вычислительной сложности алгоритма.

При построении SEMинтегралы (7-9) удобно записать в декартовой системе координат $(\xi, \eta, \zeta)$ для канонического куба $E:[-1 ; 1]^{3}$, который с помощью преобразования координат отображается на физический элемент-гексаэдр. Для примера, элемент матрицы масс запишется следующим образом:

$$
M_{i j}=\int_{\Omega} N_{i} \rho N_{j} d \Omega=\int_{E} N_{i} \rho N_{j} \cdot J d E,
$$

где $J$ - якобиан преобразования координат (Фихтенгольц 1968). Обычно в качестве отображающей функции используется трилинейная функция с 8 коэффициентами, которая 
переводит 8 вершин канонического куба в 8 вершин физического гексаэдра, при этом в силу линейности прямые ребра канонического куба переводятся в прямые ребра гексаэдра. При использовании же триквадратичной функции отображения, с 27 коэффициентами, ребра и грани элемента будут описываться квадратичными сплайнами, что позволяет намного более точно аппроксимировать геометрию модели формойэлемента (Рисунок 2). Будем называть элементы с трилинейной отображающей функцией «прямыми» или элементами HEX8, a c триквадратичной - «криволинейными» или НЕX27.

\section{Сеточные моделии результаты расчетов}

Для моделирования каротажных измерений был выбран цилиндр высотой 7 м и радиусом 3 м, вмещающий в себя упругую среду плотностью $\rho=2340 \kappa{ }^{\cdot} \mathrm{M}^{-3}$, скоростью продольной волны $V p=2733 \mathrm{~m} \cdot \mathrm{c}^{-1}$, скоростью сдвиговой волны $V s=1282 \mathrm{~m} \cdot \mathrm{c}^{-1}$. Вдоль оси цилиндра была расположена цилиндрическая скважина радиуса $0.16 \mathrm{~m}$, заполненная жидкостью плотности $\rho_{f}=1300$ кг $\cdot \mathrm{M}^{-3}$ и скоростью звука $V p=1337 \mathrm{~m} \cdot \mathrm{c}^{-1}$.Монопольный источник сигналов с центральной частотой 5 кГц расположен на оси скважины, распространяющиеся волны приходят на систему кольцевых приемников, имитирующих расположение вдоль прибора. Дисперсионные свойства волны Стоунли, получаемые по методу Ekstrom (1996), сильно зависят не только от физических свойств среды, но также от вариаций геометрии скважины.

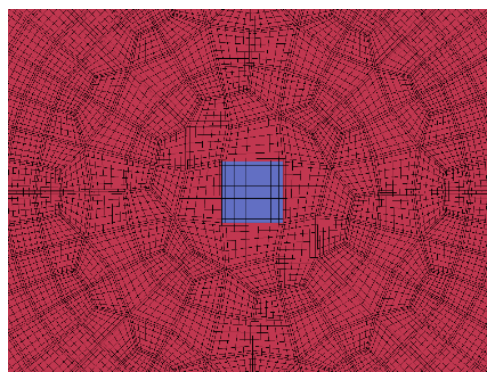

a)

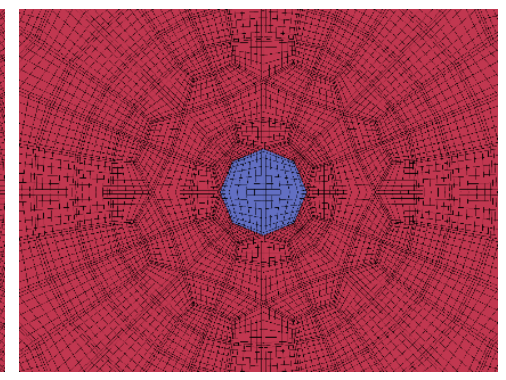

b)

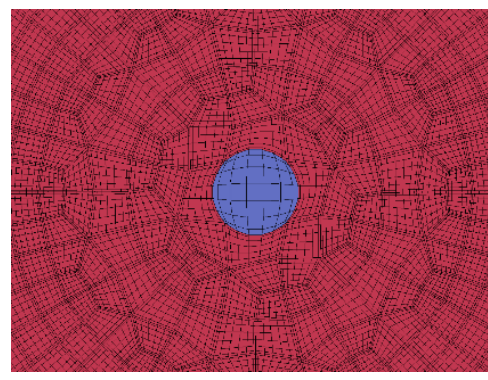

c)

Рисунок2Двумерный срез спектрально-элементной сетки 8-го порядка аппроксимации для модельной задачи акустического каротажа. Иллюстрируется изменение геометрической формы скважсины (синий ивет) в зависимости от количества узлов сетки на границе скважины. Внутри каждого четырехугольного элемента показана дополнительная сетка из $8 x 8$ узлов кубатурной формуль GLL.a) 4 узла сетки на границе, т.е. один прямой элемент на скважину; b) 8 узлов на границе; с) 4 узла на границе, один криволинейный элемент на сечение скважины, описывающий её иилиндрическую форму.

Для иллюстрации этой зависимости были построены две системы неструктурированных гексаэдральныхсеток 8-го порядка аппроксимации, геометрически сходящихся к цилиндрической форме скважины, состоящие соответственно из элементов $\operatorname{HEX} 8$ (4, 8, 16 и 32узла на границе сечения скважины) и HEX27 (4 и 8 узла на границе). Двумерные срезы некоторых сеток представлены на Рисунке 2.

Результаты обработки данных синтетических сейсмограмм приведены на Рисунке 3. В части а) показаны зависимости медленности диспергирующей волны Стоунли от частоты для четырех сеток HEX8 с разным количеством узлов на границе между скважиной и породой. Дисперсионные кривые практически неотличимы для сеток с 16 и 32 узлами на границе, что свидетельствует о достигнутой сходимости к геометрической форме скважины и неотличимости решений при данном методе анализа (Ekstrom 1996). В части b) сравниваются дисперсионные кривые для решения на HEX8-сетке с 32 узлами на границе и два решения на сетках HEX27, с 4 и 8 узлами на границе. Можно отметить сходимость решения HEX27 уже 
для 4 узлов на границе, что соответствует одному криволинейному элементу на сечении скважины.

\section{Выводы}

Метод спектральных элементов был применен для решения задачи распространения волн при акустическом каротаже. Точность решений продемонстрирована на основе дисперсионного анализа синтетических сейсмограмм, полученных на сетках HEX8 иНЕХ27. Сетки с элементами HEX8 необходимо дополнительно измельчать, чтобы приблизиться к цилиндрической форме скважины, в то время как уже одного криволинейного элемента HEX27на сечение скважины достаточно для совпадения дисперсионной кривой с результатом на подробной сетке из прямых элементов.

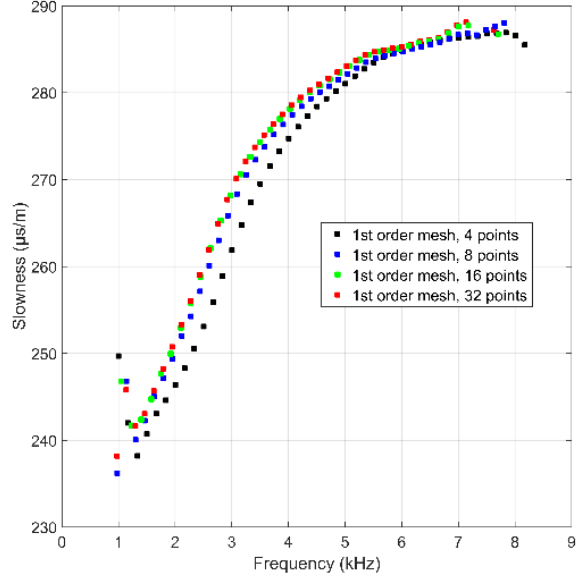

a)

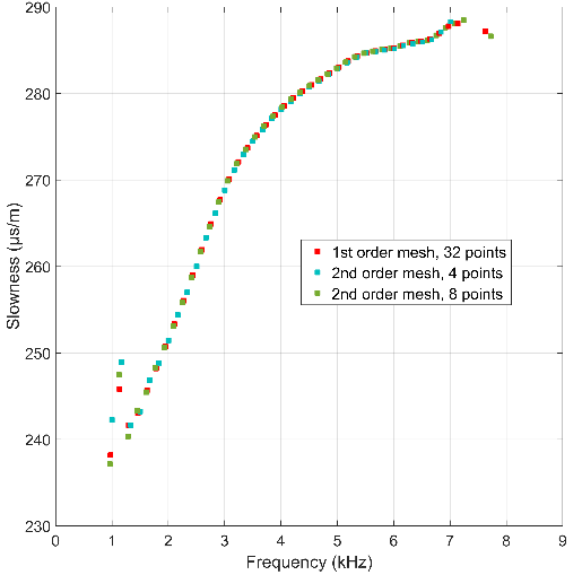

b)

РисунокЗЗависимость медленности волны Стоунли от частоты. а) Кривые для последовательности сеток из элементов НЕХ8. Для сеток с 16 и 32 точками на границе скважины данные практически совпадают (красные и зеленые маркеры), тем самым достигнута сходимость результата. b) Кривые для сеток из элементов НЕХ27, с 4 и 8 точками на границе, сравниваются с наилучшим результатом для сетки из прямых элементов,с 32 точками на границе. Сходимость достигается уже для сетки НЕХ27 с 4 точками.

Скорость работы алгоритма определяется количеством элементов в расчетной сетке и количеством шагов по времени, необходимых для получения решения в заданном временном интервале. Количество шагов по времени определяется из условия стабильности явного интегрирования уравнений (6) и обратно пропорционально зависит от минимального шага сетки. При использовании сетки HEX27 с 4 точками на границе вместо сетки HEX8 с 32 точками количество элементов уменьшается в 2 раза, с $7 \cdot 10^{4}$ до3.5 $\cdot 10^{4}$. Минимальный шаг сетки, напротив, увеличивается в 4 раза, с 0.02 м до 0.08 м. Это дает экономию ресурсов памяти и ускорение времени расчетов при одинаковой точности получаемого решения задачи. При этом использование метода высокого порядка оправдано существенной экономией компьютерной памяти на хранении малого количества ненулевых элементов матриц (7-9), что позволяет вести расчеты даже для больших трехмерных задач без использования кластеров.

\section{Библиография}

Горбачев, Ю.И. [1990] Геофизические исследования скважин. Недра, Москва. Фихтенгольц, Г.М. [1968] Основы математического анализа. Том 2. Наука, Москва. 


\section{EAGE}

Ekstrom, M.E. [1996] Dispersion estimation from borehole acoustic arrays using a modified matrix pencil algorithm. Proceedings of ASILOMAR-29.Pacific Grove, California.

Komatitsch, D., Barnes, C., Tromp, J. [2000] Wave propagation near a fluid-solid interface: A spectral-element approach. Geophysics, 65(2), pp. 623-631.

Zienkevich, O.C. and Taylor, R.F. [2000] The Finite Element Method. Volume 1. ButterworthHeinemann, Oxford.

\section{References}

Gorbachev Yu.I. [1990] Wellbore geophysical research. Nedra, Moscow.

Fichtenholz G.M. [1968] Basics of mathematical analysis. Vol 2. Nauka, Moscow.

Ekstrom M.E. [1996] Dispersion estimation from borehole acoustic arrays using a modified matrix pencil algorithm. Proceedings of ASILOMAR-29. Pacific Grove, California.

Komatitsch D., Barnes C., Tromp J. [2000] Wave propagation near a fluid-solid interface: A spectral element approach. Geophysics, 65(2), pp. 623-631.

Zienkevich O.C. and Taylor R.F. [2000] The Finite Element Method. Volume 1. ButterworthHeinemann, Oxford. 